\title{
Simple or Arthrodesis Recalibration in The Degenerative Narrow Lumbar Channel
}

\author{
Héctor Ochoa Carrillo*1, Liborio Abad Miranda ${ }^{2}$ and Rodolfo Carrillo Mora ${ }^{2}$ \\ ${ }^{1}$ Coordinator of the Orthopedic Service, Mexico \\ ${ }^{2}$ Orthopedic Service, Metropolitan Angels Hospital, Mexico
}

Submission: November 05, 2016; Published: November 17, 2016

*Corresponding author: Héctor Ochoa Carrillo Tlacotalpan 59-105 Col. Roma Sur. E-mail: hochoamx@prodigy.net.mx; Web:

wwwmchectorochoa.com.mx

\begin{abstract}
Low back pain also known as low back pain is a common reason for consultation in medical practice as it generates two million queries per year of primary care in the US. In our country due to lack of records, the number of consultations generated by this pathology is unknown. The statistics mention that only 2 out of 10 people will pass through without experiencing low back pain and that in industrialized countries three quarters of the population have suffered severe back pain. The vertebral canal can be narrow in segmental form being degenerative type or along the whole column by congenital causes. This disorder affects the area where the horse's tail and roots pass.

It is frequent between the $5^{\text {th }}$ and $6^{\text {th }}$ decade of the most non-exclusive life of this age group, since occasionally it suffers young people. The narrow duct or stenosis is a decrease of the spinal lumbar canal that exerts pressure on the horse's tail and its roots, mainly by the disc bulge towards the back, its light, decrease of the disc height, thickening of the plates, Hypertrophy of the yellow ligament, protuberances of the posterior joints, facet osteoarthritis and osteoporosis. The sagittal diameter is generally $19 \mathrm{~mm}$ from L1 to L5 and from $20 \mathrm{~mm}$ from L5 to S1, however there are individual anatomical variations according to the ethnic group in question.
\end{abstract}

Objectives

a) The concept of lumbar channel. Narrow degenerative.

b) Describe your method of study.

c) Treatment alternatives are discussed.

\section{Definition}

The narrow lumbar canal is the narrowing of the spinal canal and the lateral recesses and / or foramina through which the nerve roots pass. Therefore, they may be of the central, subarticular or extra foraminal type.

\section{Introduction}

\section{What causes the narrow passageway?}

Some patients are born with this pathology but the most frequent is that the narrow passage occurs in patients older than 50 years. In these cases, the narrow duct is the gradual result of aging and "wear" of the spine with daily activities, bad postures at work, type of sports practiced, increase in body weight that causes mechanical overload, use of heel High, weak muscle masses of the rectus abdominis; All, factors that cause alterations in the normal angles of the column. It is likely that there is a genetic predisposition to this pathology, since only a minority of people develops advanced symptomatic changes. Other causes are rheumatic diseases, degenerative joint disease and ankylosing spondylitis, among others [1].

As people age, the ligaments in the spine thicken and harden (a process called calcification). The bones and joints deform and form osteophytes. Hernias or disc protrusions are also common. Likewise, spondylolisthesis may be present, leading to compression. When these disorders occur in the area of the 
spine, they can cause the spinal canal to narrow, causing pressure on the spinal nerves and their roots causing the pain in the lower back region to be more intense and disabling as Consequence of the loss of the biomechanics of the spine. It is common for the degeneration that causes the canal to be slowed down and that the nerve structures adapt to the change, resulting in a low neurological symptomatology and mechanical presentation in most patients (Figures $1 \& 2$ ).

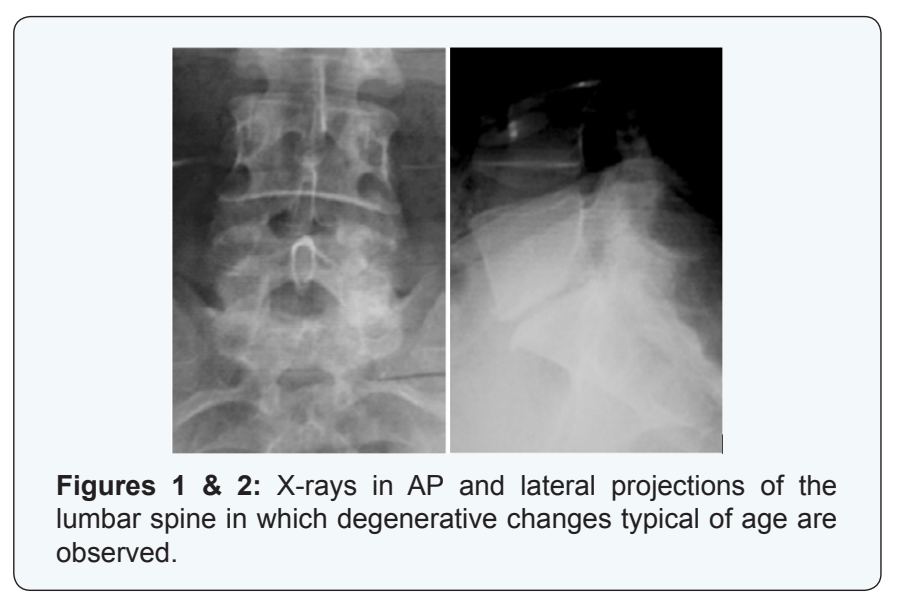

\section{How to get to the diagnosis?}

It is convenient to talk about the clinical aspect to arrive at a certain diagnosis about this pathology. It is recommended to make a medical history and a thorough exploration to evaluate the vertebral column in which the undressed patient should be examined and without footwear in order to evaluate: gait, asymmetry of the pelvis, evident deformities of the vertebral column, vices of posture , Assess: each of the nerve roots, patellar and achilles reflex, muscular strength and especially signs of neurotension, which some authors consider as positive signs of radicular compression by herniated disc.

\section{Auxiliary diagnostic studies}

It is very important to mention the studies of imaging, electrophysiology and laboratory that should be protocolized.

i. Anteroposterior, lateral, oblique and dynamic radiographs. The patient should be standing without footwear using an uncollimated 14" X 17" plate.

ii. Electromyography of pelvic limbs.

iii. Somatosensory triggered potential of pelvic limbs.

iv. Melotac can be requested.

v. Hydromyography is a study that is used in regions where there is no technological resource or the economic aspect does not allow it.

vi. And the most compelling study, magnetic resonance imaging (Figure 3).

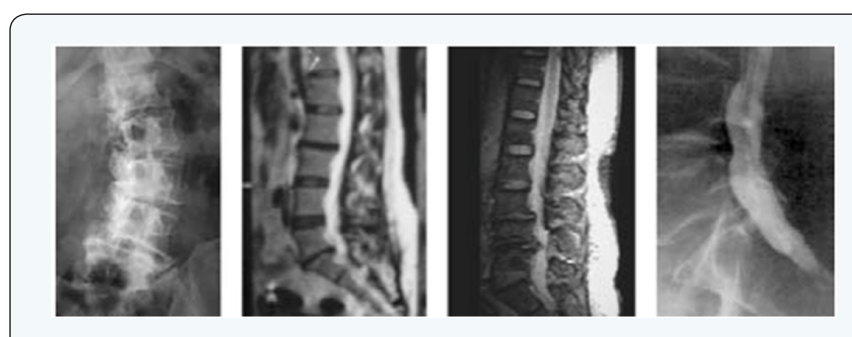

Figure 3: Note image in rosary, typical of narrow degenerative lumbar.

\section{Treatment}

The treatment of this pathology, when causing root compression, is divided into two major groups:

a. Surgical treatment: The purpose of surgery is to relieve pressure on the spinal root or nerve by enlarging the spinal canal and foramen; this is achieved by removing, trimming or realigning the affected parts that contribute to pressure.

The most common surgery in the lumbar spine is called decompressive laminectomy involves removing the laminae of the vertebrae and thus increase the space for the nerves. The surgeon can make a laminectomy with or without fusion of the vertebrae or removing the disc. You can use various devices such as pedicle screws, rods, crossbeams to enhance fusion and support unstable areas of the spine, accompanied by abundant bone graft posterolateral autologous and homologous, after extensive bone scarification and thus achieve proper integration graft. In some cases it requires $360^{\circ}$ arthrodesis [2].

Surgery should be generally indicated taking into consideration the following aspects:

i. Lumbar pain that limits physical activity.

ii. Unacceptable quality of life.

iii. Muscle weakness or numbness of the legs.

iv. Difficulty walking or standing.

b. Recalibration Technique: The following is the original technique described by Dr. Jacques Senegas, which was published in 1988 in Revue de Chirurgie Orthopedique et reparatrice de l'appareil motear [3].

The principle of re-calibration of the spinal canal consists of increasing the diameter of each of the three portions that are stenosed (dural duct, root canal, and foramen), preserving an important part of the posterior arches, as well as of the articular facets With the purpose of not destabilizing the lumbar spine. We associate this procedure automatically with dacron ligamentplasty to improve the stability of the mobile segments by reducing the amplitude of the movements. In the case of degenerative spondylolisthesis, it is necessary to perform an arthrodesis while preserving the posterior arches. 


\section{Orthopedics and Rheumatology Open Access Journal}

The preoperative evaluation should be done with standard, front, profile and dynamic images. It is necessary to carry out a systematic study of the spine that allows us to correctly evaluate the extent of the stenosis. Magnetic resonance allows a closer approximation of anatomical data and specifically a dynamic study of the stenosis, as well as a better visualization of the foramen; in addition an electromyogram and a study of somatosensory evoked potentials must be performed to have a preoperative electrophysiological base of reference.

\section{Placement of the patient}

The patient is placed in the ventral position, the thorax supported on a support, the hips flexed at right angles, supported by a support in the back. This operative position suppresses all abdominal compression and considerably reduces operative bleeding. Hypotension is desirable because it can be practiced without risk. We are in the habit of continuing decompression with the help of monitoring by monitoring the potentials triggered throughout the intervention. This technique shows the effectiveness of the decompression teaching in certain cases gain of relatively important latency times.

\section{Subsequent decompression}

It consists of a cephalic laminectomy and a partial facetectomy. The lumbar spine is approached by a medial incision. The interspinal ligaments and the hypertrophic part of the thorns are lifted with the clamp-gouge. The cephalic half of each spinous process is subsequently removed with the milling cutter, the complete removal of the cephalic half of each sheet before fully lifting the yellow ligaments including their insertion under the caudal portion of the sheets requires particular attention in order to be completed. The resection with aplomo of the articular facets of the pedicles carries this same operative procedure. The joint capsule is also carefully excised from the inside of the duct.

As a result of this operative time, a great decompression of the dura mater is made, as well as of the roots in each level, preserving the posterior arch, the caudal half of the lamina and the spiny. Anterior Decompression If there is a protruding disc herniation, a discectomy should be performed at this level; however, if there is only a disc bulge we prefer to treat the disc that is involved in the compression by a chemonucleolysis. This procedure has the advantage of respecting the posterior vertebral ligament as well as the posterior part of the discs involved in vertebral stabilization. If there are later body protuberances we do not remove them but we hit them with the help of a special "reductor", which is a fast and very effective procedure.

\section{The apical facetectomy}

It is the essential moment of decompression of the foramen; however, even if there is radiculopathy, it is not essential to do it at all levels and only takes place in the compromised site. Magnetic resonance imaging is precise to evaluate the elements of lateral compression. With the help of frappe scissors, the upper joint is cut horizontally and later extracted with the disc clamp. Later, it is verified that there is no lateral hernia that can be discectomized or can be treated with nucleolysis.

\section{Adipoplasty}

With the intention of limiting the postoperative fibrosis around the dura and the roots we performed an adipoplasty at each decompressed level. With the purpose of reducing operative time and avoiding compression by the adipose grafts, we subtract through the same incision, on the patient's side, a long greasy layer to allow the passage of the spinous processes. The stabilization of the decompressed area In order to prevent the recurrence of degenerative lesions and therefore low back pain, it is essential to stabilize the decompressed area. When there is no spondylolisthesis we systematically perform an interspinous ligamentplasty with the aid of a flexible and flat dacron ligament passed eight times around the spiny and sutured with a point in the interspinous space. This procedure has been experimented in the laboratory and allows limiting $15 \%$ the amplitude of the movements of the lumbar spine.

In the case of degenerative spondylolisthesis, the recalibration of the lumbar duct, due to the preservation of the posterior arches, allows the reduction and osteosynthesis to be carried out thanks to the Luque bars by means of two fixed curved metallic threads on each sheet. A posterolateral bone graft is applied. If the spondylolisthesis cannot be reduced, it is necessary to completely remove the lamina of the displaced vertebra by performing the same procedure of fixation and arthrodesis. After the operation, the patient is placed in a molded polyethylene lumbar orthosis over a period of three months.

\section{Other treatment alternatives}

Conservative treatment Alternatives with which the narrow lumbar canal can be treated conservatively:

I. Medications, including non-steroidal anti-inflammatory drugs (NSAIDs), to reduce inflammation and pain, as well as analgesics.

II. Corticosteroid injections (epidural steroids) help reduce inflammation and decrease acute pain that extends to the hips or leg. This pain relief may be only temporary. It is recommended that no more than 3 injections be applied within 6 months (therapeutic blocks).

III. Anti-neuritic medication.

IV. Weight control.

V. Rest or restriction of activity (this may vary depending on the extent of neurological involvement).

VI. Physiotherapy and / or prescribed exercises to help stabilize the spine, increase endurance and flexibility.

VII. Column hygiene. 
VIII. Swimming (which in addition to strengthening the muscles is anti-stress).

IX. Use of acute phase orthoses only. Conservative management should be done for two to three months.

When this resource is exhausted, it has the alternative of surgical management, which should have as its main objective to relieve pain and reintegrate the person into their daily activities.

\section{Our Experience}

The surgical behavior that we have carried out during the last years is:

\section{Laminectomy expands}

It consists of the extirpation of the laminae, part of the facet processes to enlarge the stenosed lumbar canal, exposing the nervous tissue in the affected levels, checking the integrity of the dura mater, nerve roots on each side to ensure that any pressure on Them and the equine tail. Finally bone fragments and soft tissues are removed as yellow ligament and disc herniation. This technique is recommended in patients over 60 years of age in whom, by nature, the anterior region of the spine is fused due to anterior longitudinal ligament calcification, osteophytes and deformity of the vertebral bodies giving the image in the parrot beak. It has been demonstrated that a less rigid fusion such as that achieved by an uninsulated arthrodesis produces less segmental degeneration, in addition to that a posterolateral arthrodesis in which no bone bridge development is observed in the subsequent radiographs of control produces a sufficient fibrous union for the Stabilization of the segment, resulting in significant clinical improvement (Figure 4) [4,5].

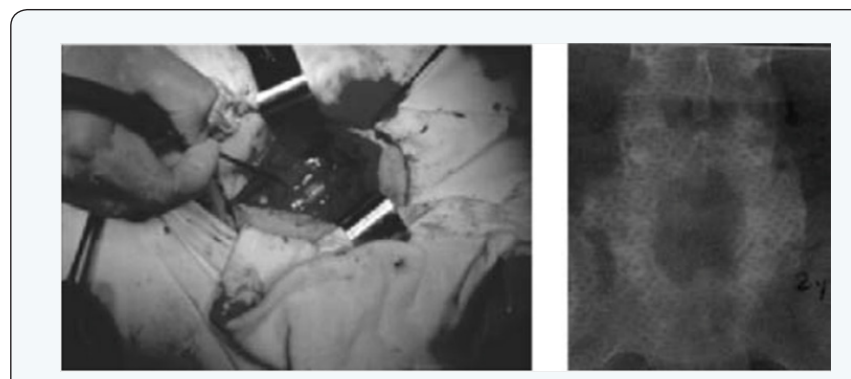

Figure 4: Arthrodesis without instrumentation.

\section{Wide laminectomy plus posterolateral arthrodesis}

Considering the above concept, an unstable spine (WithePanjabi) is left biomechanically, in order to avoid this it is necessary to apply the resource of posterolateral arthrodesis with abundant mixed bone graft (autologous-homologous), after extensive scarification of the transverse processes of the levels Intervened and give opportunity for proper bone integration and thus have the desired stability secondarily. This technique is recommended in patients who are in the 5th decade of life (Figure 5).

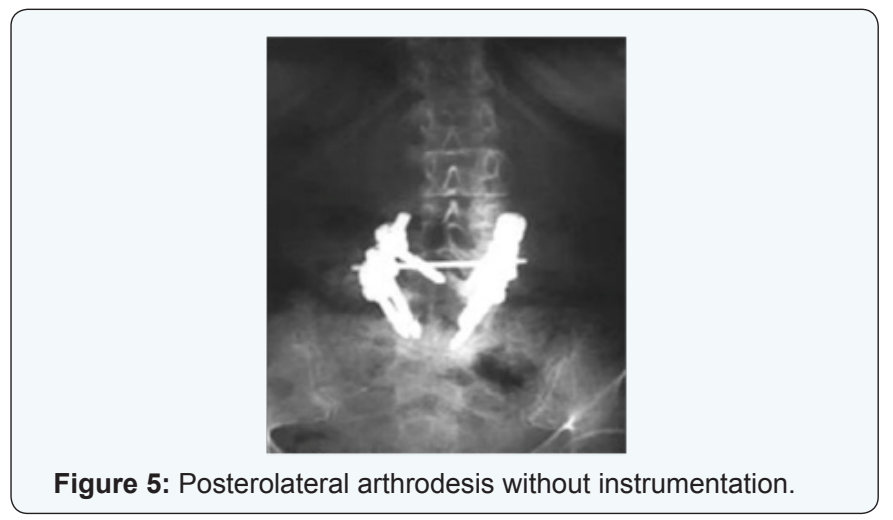

Wide laminectomy with instrumentation + posterolateral arthrodesis

When performing a broad laminectomy, it is necessary to consider - according to the radiographic findings, magnetic resonance, patient's age, physical conditions, as well as its activity and trans-operative assessment - which, in addition to posterolateral bone grafting, must be considered with consistent instrumentation In 2 bars, transpedicular screws and a crossbar to give it more support and stability (Figures $6 \& 7$ ).

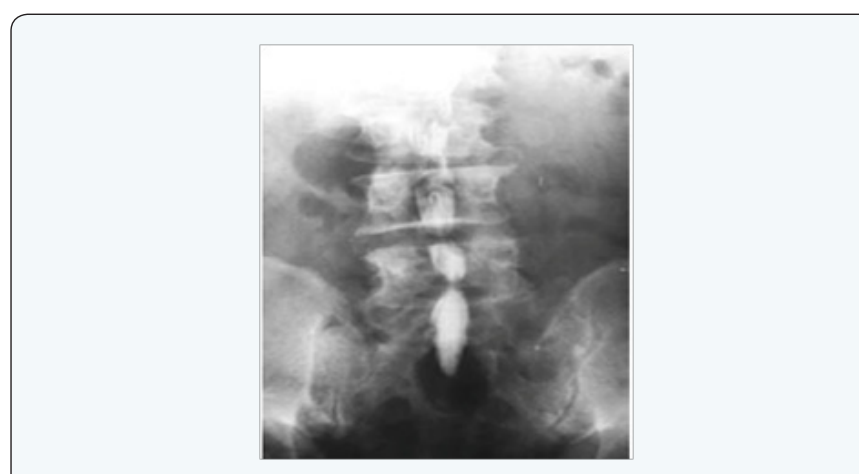

Figure 6: Posterolateral arthrodesis with instrumentation through bars, transpedicular screws and crossbar.

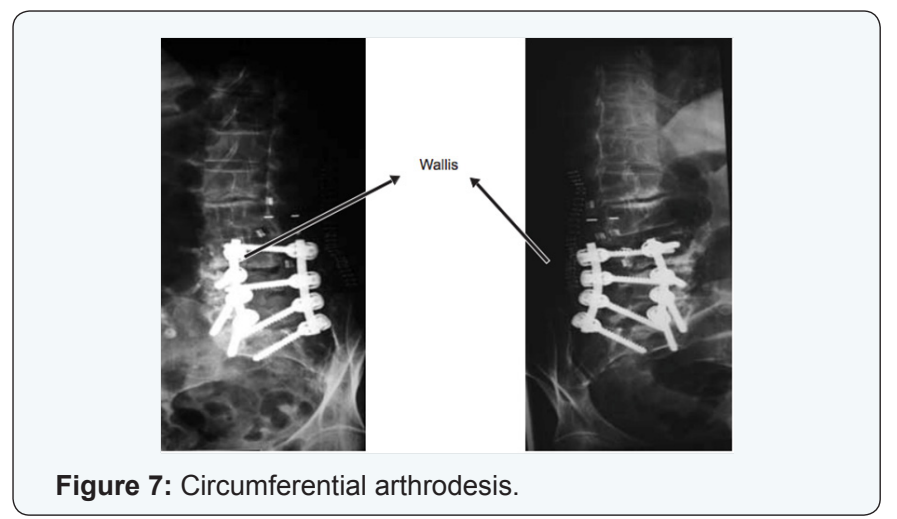

Wide laminectomy with instrumentation $+360^{\circ}$ arthrodesis

Occasionally there will be patients with severe spinal instability who, in addition to the previous procedure, will be added $360^{\circ}$ arthrodesis (circumferential arthrodesis) to obtain maximum clinical and biomechanical benefit using intersomatic 
bone, cages, boxes, intersomatic spacers and finally, Posterior instrumentation with bars fixed to transpedicular screws, hooks, et cetera.

\section{Conclusion}

The last biomechanical analyzes have concluded that in doing some of these procedures, over time, instability of the overlying segment is generated, which is why it is recommended to use a semi-rigid semi-rigid Wallis or dynamic DIAM type spacer. In our service, as of November 2005, a study protocol is being developed focused on the analysis of the dynamic spacer which is placed in the overlying level in patients submitted to root release accompanied by arthrodesis and instrumentation in the lower segments [6-15].

\section{References}

1. Gelbard A (2014) Causes and Consequences of Laryngotracheal Stenosis. The Laryngoscope.

2. Ricketti PA, Ricketti AJ, Cleri DJ, Seelagy M, Unkle DW et al. (2010) A 41-year-old male with cough, wheeze, and dyspnea poorly responsive to asthma therapy. Allerg Asthma Proc 31(4): 355-358.

3. Parrish RW, Banks J, Fennerty AG (1983) Tracheal obstruction presenting as asthma. Postgrad Med J 59(698): 775-776.
4. Galvin IF, Shepherd DR, Gibbons JR (1990) Tracheal stenosis caused by congenital vascular ring anomaly misinterpreted as asthma for 45 years. Thorac Cardiovasc Surg 38(1): 42-44.

5. Kokkonouzis I, Mermigkis C, Psathakis K, Tsintiris K (2005) Postintubation tracheal web. J Broncho 12(4): 271-272.

6. Legasto AC, Haller JO, Giusti RJ (2004) Tracheal web. Pediatr Radiol 34(3): 256-258.

7. Nguyen NK (2006) Unexpected tracheal web encountered during difficult intubation in the operating room. Proc (Bayl Univ Med Cent) 19(3): 224-225.

8. Al Badaai Y, Nguyen LH (2008) Difficult intubation due to undiagnosed congenital tracheal web. Int J Ped Otorhinolaryngology 72(9): 14371439.

9. Egawa A, Hirabayashi Y, Seo N (2011) Case of unexpected difficult intubation caused by asymptomatic congenital laryngeal web. Masui 60(10): 1211-1213.

10. Al-Qadi MO, Artenstein AW, Braman SS (2013) The forgotten zone: Acquired disorders of the trachea in adults. Respir Med 107(9): 13011313.

11. Empey DW (1972) Assessment of upper airways obstruction. Br Med J 3(5825): 503-505.

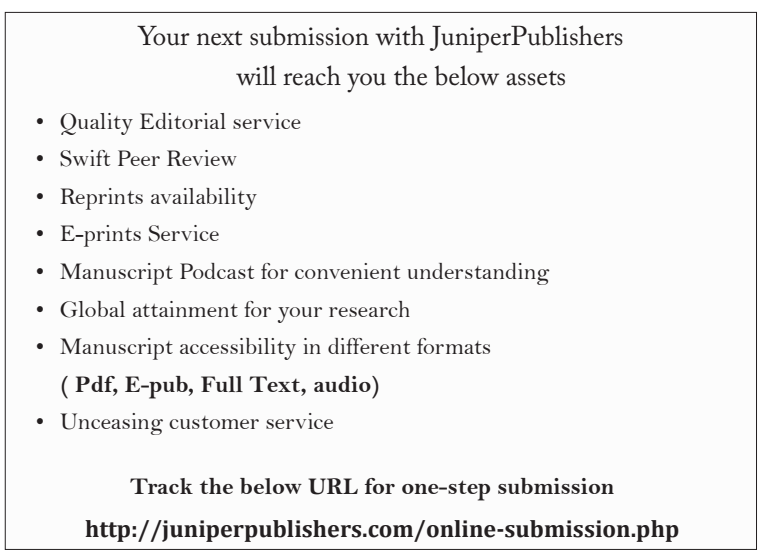

